\title{
Insulin, Regular
}

National Cancer Institute

\section{Source}

National Cancer Institute. Insulin, Regular. NCI Thesaurus. Code C29125.

A recombinant form of the naturally occurring human pancreatic hormone insulin. Upon administration, regular insulin mimics the action of endogenous human insulin and binds to insulin receptors located on muscle and fat cells. This both facilitates the cellular uptake of glucose and lowers blood glucose levels. In addition, insulin inhibits the liver's conversion of stored glycogen into glucose, which also decreases blood glucose levels. Insulin also inhibits lipolysis in adipose tissue, inhibits proteolysis, and enhances protein synthesis. 